\title{
Latexin exhibits tumor suppressor potential in hepatocellular carcinoma
}

\author{
QING-FENG NI, YUAN TIAN, LIANG-LIANG KONG, YE-TING LU, WEN-ZHOU DING and LIAN-BAO KONG \\ The First Affiliated Hospital of Nanjing Medical University, The National Institute of Living Donor Liver Transplantation, \\ Nanjing, Jiangsu 210029, P.R. China
}

Received November 13, 2013; Accepted December 16, 2013

DOI: $10.3892 /$ or.2014.2966

\begin{abstract}
Hepatocellular carcinoma (HCC) is the most common primary cancer of the liver and latexin is downregulated in several types of human cancer. However, latexin expression in $\mathrm{HCC}$ remains unknown. mRNA expression of latexin in HCC samples and HCC-derived cell lines was detected by semi-quantitative PCR and real-time PCR, while protein expression was assessed by immunohistochemistry. The role of latexin in the regulation of the proliferation of HCC-derived cells was investigated both in vitro and in vivo. Flow cytometry was used to differentiate cell cycle distribution in SK-hep-1 and YY-8103. In a total of 60 paired HCC specimens, compared with adjacent non-cancer tissues, latexin mRNA was downregulated in 42 specimens. Immunohistochemical analysis showed a significant reduction in latexin expression in HCC compared to control tissues. Overexpression of latexin inhibited SK-hep-1 and HepG2 cellular colony formation and tumor growth. Conversely, YY-8103 and Focus cells transfected with shRNA enhanced colony formation and tumor growth. Latexin overexpression promoted cell cycle arrest in the G0/G1 phase in SK-hep-1 and silencing of latexin promoted the cell cycle transition from G0/G1 phase to S phase in YY-8103. The cyclin-dependent kinase inhibitors (CDKIs) (p21Cip1, p27Kip1, p15INK4B), cyclin D1 and cyclin E were shown to be differentially expressed in latexin-overexpressed cells and latexin-silenced cells. These results indicated that latexin may be an effective target for gene therapy.
\end{abstract}

\section{Introduction}

Hepatocellular carcinoma (HCC) is the most common primary cancer of the liver and the third most frequent cause

Correspondence to: Dr Lian-Bao Kong, Department of Liver Transplantation Center, The First Affiliated Hospital of Nanjing Medical University, The National Institute of Living Donor Liver Transplantation, 300 Guangzhou Road, Nanjing, Jiangsu 210029, P.R. China

E-mail: lbkong@njmu.edu.cn

Key words: latexin, hepatocellular carcinoma, proliferation, cell cycle of cancer-related mortality in the world, with more than 660,000 deaths per annum (1-5). The major etiologic factors of $\mathrm{HCC}$ are hepatitis $\mathrm{B}$ virus (HBV) and hepatitis $\mathrm{C}$ virus (HCV) infection and various other non-viral-related causes of liver cirrhosis (6). Over the past few decades, the incidence of HCC has increased in eastern Asia and sub-Saharan Africa $(7,8)$. Surgery currently offers the only possibility of prolonged survival in HCC patients. However, recurrence occurs in more than two-thirds of these patients despite initial curative intent, and, thus, HCC has a dismal prognosis $(9,10)$. Therefore, it is vital to identify new clues to understand the pathogenesis of HCC and to explore effective therapeutic strategies.

The latexin gene mapped to chromosome $3 \mathrm{q} 25.32$ was originally identified in the lateral neocortex of rats and serves as a marker of regionality and development in the rodent nervous system (11). The human latexin gene encodes the latexin protein comprised of 222 amino acids with $84.2 \%$ identical to rat and $84.7 \%$ identical to mouse latexin proteins (12). It consists of two topologically equivalent subdomains, each one with a cystatin-like topology, consisting of an $\alpha$-helix enveloped by a curved $\beta$-sheet. These subdomains are packed against each other through the helices and linked by a connecting segment encompassing a third $\alpha$-helix $(13,14)$. Latexin has been reported to act as an endogenous vertebrate carboxypeptidase inhibitor (CPI). However, its sequence is unrelated to any other reported CPIs, but shows significant homology with the putative tumor suppressor, tazarotene-induced gene 1 (TIG1), suggesting a familial relationship $(13,15)$. Liang et al revealed that latexin functions in the negative control of hematopoietic stem cell (HSC) populations in mice by decreasing cell replication and increasing apoptosis (16). Latexin-deficient HSCs have been shown to possess an enhanced colony-forming ability (17). Elevated latexin expression has also been reported in normal human stem cells compared to the same cell populations from patients with acute myelogenous leukemia (AML) or lymphoma. The ectopic expression of latexin in mouse lymphoma cells lacking latexin expression shows marked suppression of growth in vitro (18). A study by Ke et al identified high levels of latexin expression in an immortalized human gastric epithelial cell line, GES-1, as compared to expression in the MC cell line, which is the malignant derivative of the GES-1 cell line (19).

The cell cycle progression pathways are the endpoint of signaling cascades implicated in cell growth and cell prolif- 
eration. The cell cycle is tightly coordinated by sequential assembly and activation of phase-specific protein kinase complexes $(20,21)$ formed by cyclins and cyclin-dependent kinases (CDKs), which are also regulated by the INK4 proteins and the CDK inhibitors (CDKIs). D-type cyclins are expressed throughout the cycle in response to mitogen stimulation (21). Cyclin D-CDK4 and cyclin E-CDK2 complexes are required for the passage from $\mathrm{G} 1$ to $\mathrm{S}$ phase. The levels of CDKI are high in quiescent cells, fall in response to mitogenic stimulation, remain at threshold levels in proliferating cells and increase again when mitogens are withdrawn (21).

In the present study, we found that latexin was markedly downregulated in HCC specimens, compared to adjacent non-cancerous tissues. This indicated that latexin may contribute to the inhibition of cellular proliferation in HCC. The experiments showed that overexpression of latexin inhibited HCC-derived SK-hep-1 and HepG2 cellular proliferation in vitro and vivo. In contrast, latexin knockdown via shRNA markedly promoted these phenotypes in YY-8103 and Focus cells. Furthermore, the mechanistic analyses indicated that latexin influenced cell cycle transition by modulating the quantities of cell cycle regulators.

\section{Materials and methods}

Tissue specimens. To examine the expression of latexin in human tumor tissues, a total of 60 paired HCC and adjacent non-tumor tissues were used in the present study. Adjacent non-tumor tissues were removed $2 \mathrm{~cm}$ away from the edge of the primary tumors. Both HCC specimens and adjacent non-tumor tissues were confirmed by pathological examination and immediately stored in liquid nitrogen after surgery. The study protocols for the investigations involving human tissue, or animals, were approved by the Institutional Animal Care and Use Committee at Nanjing Medical University.

Liver cancer cell lines. The human HCC cell lines (YY-8103, Focus, LM3, LM6, HepG2, Hep3B, SK-hep-1, Huh-7, MHCC-97H, MHCC-97L, SMCC-7721) were obtained from the Department of Liver Transplantation Center, The First Affiliated Hospital of Nanjing Medical University. These cell lines were propagated in a $5 \% \mathrm{CO}_{2}, 37^{\circ} \mathrm{C}$-humidified incubator and cultured in Dulbecco's modified Eagle's medium (DMEM) containing $10 \%$ fetal bovine serum (FBS), penicillin $(50 \mathrm{U} / \mathrm{ml})$ and streptomycin $(50 \mu \mathrm{g} / \mathrm{ml})$.

Semi-quantitative RT-PCR and quantitative real-time PCR (qRT-PCR). Total RNA from tissue samples and cultured cells was extracted using TRIzol reagent (Invitrogen) and then reverse transcribed into cDNA using the PrimeScript RT reagent kit (Takara). qRT-PCR assays were carried out to detect mRNA expression using SYBR Premix Ex Taq (Takara) according to the manufacturer's instructions. The latexin primers used in qRT-PCR were: forward primer, 5'-CCTGGGTTGCCTGTGGTTAT-3' and reverse primer, 5'-CTTCCTTTGGCAGACGGCTA-3'. $\beta$-actin was used as an internal control and amplified with forward primer, 5'-AGAGCCTCGCCTTTGCCGATCC-3' and reverse primer, 5'-CTGGGCCTCGTCGCCCACATA-3'.
Immunohistochemical staining. All tissues were paraffinembedded and were obtained from the Department of Pathology, The First Affiliated Hospital of Nanjing Medical University. The paraffin-embedded tissues were cut into $4-\mu \mathrm{m}$ sections and then incubated with rabbit anti-latexin polyclonal antibody (1:200; Abcam) at $4^{\circ} \mathrm{C}$ overnight, SP-9000 Histostain ${ }^{\mathrm{TM}}$-Plus kits (ZSGB-BIO) were used according to the manufacturer's protocol. Scoring was measured by the cell cytoplasm staining pattern: score of 0 , absent cell cytoplasm staining; score of 1 , weak cell cytoplasm staining; score of 2 , moderate cell cytoplasm staining; and score of 3 , strong cell cytoplasm staining.

shRNA preparation. Two siRNAs against latexin were designed according to the web of Invitrogen Co. and chemically synthesized by Shanghai GenePharma Co. (Shanghai, China). The sequence of siRNA-424 was: GCACAGCUGAA GUACUUUAdTdT (sense) and UAAAGUACUUCAGCUGU GCdTdT (antisense). The sequence of siRNA-444 was: CCU UCAACGGGACAAGAAAdTdT (sense) and UUUCUUGU CCCGUUGAAGGdTdT (antisense). Negative control (NC) siRNA synthesized by Shanghai GenePharma Co. was used as a control. The sequence of si-NC was: UUCUCCG AACGUGUCACGUTT (sense) and ACGUGACCGUUCGG AGAATT (antisense).

shRNA duplexes against latexin were designed according to the web of Invitrogen Co. and synthesized by GenePharma Co. The sequences were incorporated into the vector $\mathrm{p}$-SUPER to generate p-SUPER-shRNA-latexin (GenePharma Co.). The sequence of shRNA-424 was as follows: GATCCCCGCACA GCTGAAGTACTTTATTCAAGAGATAAAGTACTTCAG CTGTGCTTTTTGGAAA (sense) and AGCTTTTCCAAAA AGCACAGCTGAAGTACTTTATCTCTTGAATAAAGTA CTTCAGCTGTGCGGG (antisense). The sequence of shRNA-444 was as follows: GATCCCCCCTTCAACGGGA CAAGAAATTCAAGAGATTTCTTGTCCCGTTGAAGGT TTTTGGAAA (sense) and AGCTTTTCCAAAAACCTTCA ACGGGACAAGAAATCTCTTGAATTTCTTGTCCCGTT GAAGGGGG (antisense). The sequence of sh-NC was as follows: GATCCCCTTCTCCGAACGTGTCACGTTTCAA GAGAACGTGACACGTTCGGAGAATTTTTGGAAA (sense) and AGCTTTTCCAAAAATTCTCCGAACGTGTCACGTT CTCTTGAAACGTGACACGTTCGGAGAAGGG (antisense). The constructs were verified by sequencing.

Construction of latexin expression vector. The latexin open reading frame (ORF) was amplified from the human liver cDNA library (Genbank: NM_020169.3) using PrimeStar PCR and constructed into the expression vector pcDNA3.1B to generate pcDNA3.1B-latexin. The sequence of the forward primer was: 5'-EcoRI-AGAGAATTCATGGAAATC CCGCCGACCAAC-3' and reverse primer, 5'-BamHI-AGAG GATCCTTATTCCAGTTGTACTTCCTTTGGC-3'. The construct was verified by sequencing.

Cell transfection. Both the shRNA and latexin expression vector were transfected using Lipofectamine ${ }^{\circledR} 2000$ (Invitrogen) according to the manufacturer's instructions.

Cell proliferation assay. Cells were seeded at a density of 2,000-5,000 cells/well in $100 \mu \mathrm{l}$ complete medium in 
96-well plates. The Cell Counting Kit-8 (CCK-8; Dojindo Laboratories) was used to measure cell viability according to the manufacturer's instructions. Each experiment was repeated at least 3 times.

Colony formation assay. To examine the effect of upregulated or downregulated latexin expression on proliferation of HCC cell lines, cells transfected with different plasmids were used for the colony formation assay. Each type of cell was seeded into $10 \mathrm{~cm}$ plates $(50,000$ cells/well) and cultured for 3 weeks in medium containing $1,000 \mu \mathrm{g} / \mathrm{ml} \mathrm{G} 418$. These cultures were stained with $0.4 \%$ crystal violet. Clones $>2 \mathrm{~mm}$ were counted and the number of clones per well was averaged from 3 wells for each experiment. Each experiment was repeated at least 3 times.

Soft agar colony formation assay. Cells transfected with different plasmids were suspended in $0.5 \mathrm{ml} 1 \%$ low melting point agarose with complete culture medium, and then layered on top of $0.5 \mathrm{ml} 2 \%$ low melting agarose in 24-well plates. Cell numbers varied from 2,000-5,000 for different cell lines. The plates were incubated in a $5 \% \mathrm{CO}_{2}, 37^{\circ} \mathrm{C}$-humidified incubator for 2 weeks. Colonies in at least 6 random microscopic fields were counted and photographed. All experiments were repeated 3 times.

Flow cytometric analysis of cell cycle. For the cell cycle assay, cells were trypsinized and rinsed twice with ice-cold PBS solution, then added to $75 \%$ ice-cold ethanol while vortexing, followed by incubation on ice for $60 \mathrm{~min}$. The fixed cells were washed with ice-cold PBS and incubated at $37^{\circ} \mathrm{C}$ for $30 \mathrm{~min}$ in $0.5 \mathrm{ml}$ PBS solution containing $20 \mathrm{mg} / \mathrm{ml}$ RNase A, $0.2 \%$ Triton X-100, $0.2 \mathrm{mM}$ EDTA and $20 \mathrm{mg} / \mathrm{ml}$ of propidium iodide. The percentage of cells in G0/G1, S and G2/M phases was determined using the Beckman Gallios Flow Cytometer (Beckman Coulter).

Tumorigenicity assay in nude mice. Male BALB/c nude mice (3-4 weeks old) were purchased from the Department of Laboratory Animal Center, Nanjing Medical University. Cells with differential latexin expression were injected subcutaneously into the lateral root of the anterior limb of nude mice (500x $10^{6}$ cells/mouse, 7 mice in each experimental group). Tumor size was measured every third day after injection. Three weeks after injection, the mice were sacrificed and photographed. Tumor weight was then calculated.

Western blot analysis. Cell lysates were prepared using cold lysis buffer containing $25 \mathrm{mmol} / \mathrm{l}$ Tris- $\mathrm{Cl}(\mathrm{pH} 7.5), 5 \mathrm{mmol} / \mathrm{l}$ EDTA, $1 \%$ SDS and protease inhibitor cocktail (Sigma). After boiling for $5 \mathrm{~min}$, samples were subjected to electrophoresis in $10 \%$ SDS-PAGE and transferred onto a polyvinylidene difluoride (PVDF) membrane and blocked for $1 \mathrm{~h}$ at room temperature with 5\% blocking buffer. The membranes were washed 3 times with $0.1 \%$ Tris-buffered saline with Tween-20 (TBST) and incubated with the primary antibody overnight at $4^{\circ} \mathrm{C}$. The membranes were washed again and then incubated with the secondary antibody at room temperature for $1 \mathrm{~h}$. Primary antibodies used in the present study included: rabbit anti-latexin polyclonal antibody (1:500), rabbit anti-p21Cip1 polyclonal antibody $(1: 1,000)$, rabbit anti-p27Kip1 polyclonal antibody (1:1,000), rabbit anti-p15INK4B polyclonal antibody $(1: 1,000)$, rabbit anti-cyclin D1 polyclonal antibody $(1: 1,000)$, rabbit anti-cyclin E polyclonal antibody (1:200) and rabbit anti- $\beta$-actin polyclonal antibody $(1: 1,000)$ (all from Abcam). Proteins were detected using an ECL western blotting detection system (Pierce) by enhanced chemiluminescence.

Statistical analysis. Statistical analysis was performed using SPSS 18.0 and Graphpad Prism 5.0 software. Quantitative data were recorded as means $\pm \mathrm{SD}$. Differences between 2 groups were assessed by Student's t-test (two-tailed). $\mathrm{P}<0.05$ was considered to indicate a statistically significant difference.

\section{Results}

Latexin expression is decreased in HCC and HCC-derived cell lines. Semi-quantitative RT-PCR and qRT-PCR were performed to measure the latexin mRNA expression levels in HCC and adjacent non-cancerous livers from 60 patients. The results indicated that latexin mRNA was decreased in $42(70 \%)$ of 60 HCC specimens compared with the matched normal liver tissues (Fig. 1A and B). We performed immunohistochemistry (IHC) to evaluate latexin protein expression in HCC specimens and paired normal liver tissues in the same 60 matched samples. Of these specimens, 45/60 (75.0\%) of cancerous specimens showed no or weak (+/-) positive staining, whereas $24 / 60(40.0 \%)$ of non-HCC tissues showed no or weak (+/-) positive staining (Fig. 1D). Furthermore, we evaluated the expression of latexin in 11 HCC-derived cell lines using qRT-PCR. Latexin mRNA was significantly decreased in all HCC-derived cell lines (YY-8103, Focus, LM3, LM6, HepG2, Hep3B, SK-hep-1, Huh-7, MHCC-97H, MHCC-97L, SMCC7721) compared with normal human liver cells (L02) (Fig. 1C). These data showed that latexin is decreased in HCC.

Overexpression of latexin inhibits proliferation and colony formation in SK-hep-1 and HepG2 cell lines. To overexpress latexin, the recombinant pcDNA3.1B-latexin was transfected into SK-hep-1 and HepG2 cell lines. We performed western blotting to evaluate latexin protein expression in SK-hep-1 and HepG2 cells transfected with pcDNA3.1B-latexin $48 \mathrm{~h}$ post-transfection. Latexin protein expression in pcDNA3.1Blatexin-transfected cells was significantly higher than in empty vector-transfected cells (Fig. 2A and B). To investigate the antiproliferative effects in pcDNA3.1B-latexin-transfected cells, cellular growth was monitored for 6 days. The pcDNA3.1Blatexin-transfected SK-hep-1 and HepG2 cells (Fig. 2C and D) showed a significant decrease in cellular growth compared with empty vector-transfected cells ( $\mathrm{P}<0.05)$. SK-hep-1 and HepG2 cells with overregulated latexin expression were subjected to colony formation assay. As shown in Fig. 2E and F, overexpression of latexin in SK-hep-1 and HepG2 cells resulted in significant inhibition of colony formation as compared with SK-hep-1 and HepG2 cells transfected with empty vector $\left({ }^{*} \mathrm{P}<0.05\right)$ and the majority of clones were smaller than those of control cells. We then used a soft agar assay for colony formation, which is the most stringent assay for detecting the proliferative ability of cells. We observed reduced formation of colonies in soft agar (Fig. 2G and H) that had been seeded with 

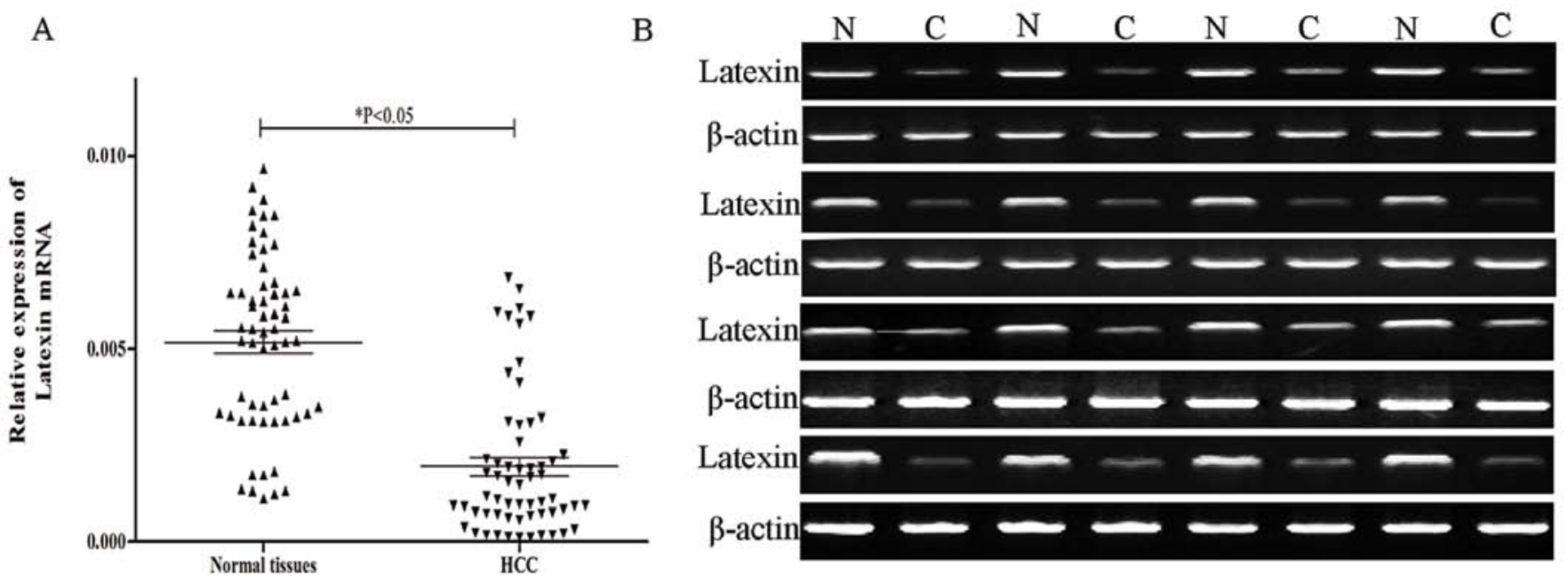

$\mathrm{C}$

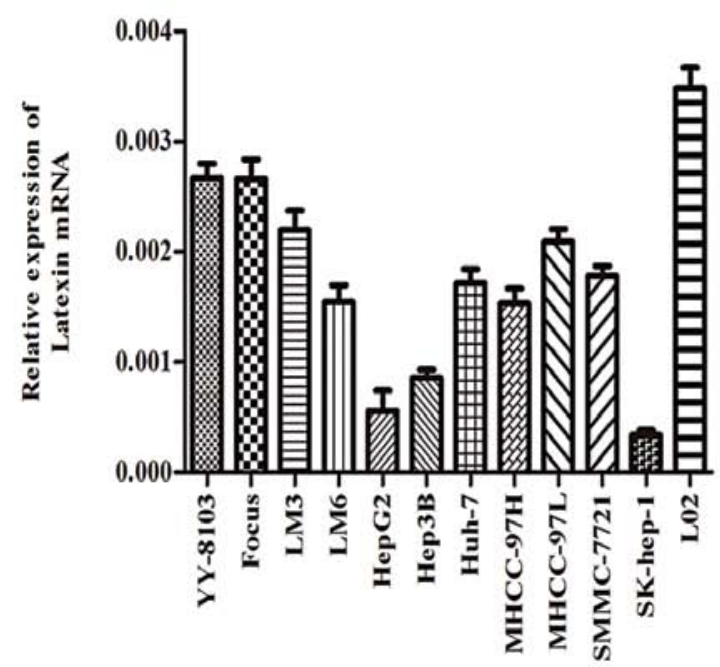

$\mathrm{D}$

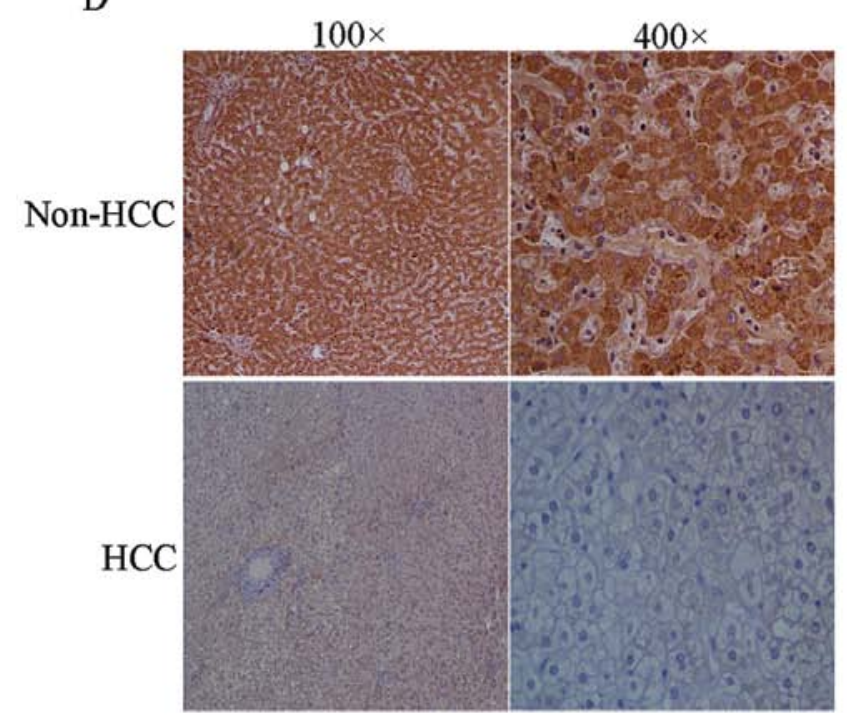

Figure 1. Downregulation of latexin in clinical specimens and HCC-derived cell lines. (A) The expression of latexin mRNA in 60 paired HCC specimens by real-time PCR ("P<0.05). (B) Representative results of the downregulation of latexin mRNA in HCC specimens by semi-quantitative RT-PCR. (C) Latexin mRNA was downregulated in 11 HCC-derived cell lines. (D) Representative results of the downregulation of latexin protein in HCC specimens by immunohistochemistry (x100 and x400 magnification). HCC, hepatocellular carcinoma.

SK-hep-1 and HepG2 cells infected with pcDNA3.1B-latexin compared with empty vector-transfected cells $\left({ }^{*} \mathrm{P}<0.05\right)$. These results indicated that latexin acts as an inhibitor of tumor cell growth in vitro.

Knockdown of latexin promotes proliferation and colony formation in YY-8103 and Focus cell lines. To knock down latexin, the recombinant p-SUPER-shRNA-latexin was transfected into YY-8103 and Focus cell lines. Western blot analyses were performed to assess the efficiency of latexin knockdown in YY-8103 and Focus cells transfected with p-SUPER-shRNAlatexin $48 \mathrm{~h}$ post-transfection. Latexin protein expression in p-SUPER-shRNA-latexin-transfected cells was significantly lower than that in sh-NC-transfected cells (Fig. 3A and B). The p-SUPER-shRNA-latexin-transfected YY-8103 and Focus cells (Fig. 3C and D) showed significant promotion of cellular growth compared with sh-NC-transfected cells $\left({ }^{*} \mathrm{P}<0.05\right)$. YY-8103 and Focus cells with downregulated latexin expres- sion were subjected to colony formation assay. As shown in Fig. 3E and F, decreased expression of latexin in YY-8103 and Focus cells resulted in significant promotion of colony formation as compared with cells transfected with sh-NC $\left({ }^{*} \mathrm{P}<0.05\right)$. We also observed enhanced formation of colonies in soft agar (Fig. 3G and $\mathrm{H}$ ) that had been seeded with YY-8103 and Focus cells transfected with p-SUPER-shRNA-latexin compared with sh-NC-transfected cells $\left({ }^{*} \mathrm{P}<0.05\right)$.

Overexpression of latexin promotes cell cycle arrest in $G 0 / G 1$ phase in SK-hep-1 cells and knockdown of latexin promotes the cell cycle transition from G0/G1 to $S$ phase in YY-8103 cells. To study the growth suppression effect of pcDNA3.1Blatexin on SK-hep-1 cells and the growth enhancement effect of p-SUPER-shRNA-latexin on YY-8103 cells, we performed cell cycle distribution analysis using flow cytometry $24 \mathrm{~h}$ after transfection. As shown in Fig. 4A and B, overexpression of latexin induced cell cycle arrest in G0/G1 phase in SK-hep-1 
A

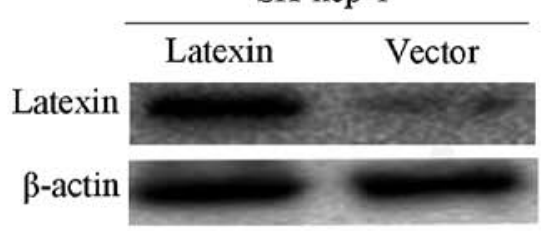

C

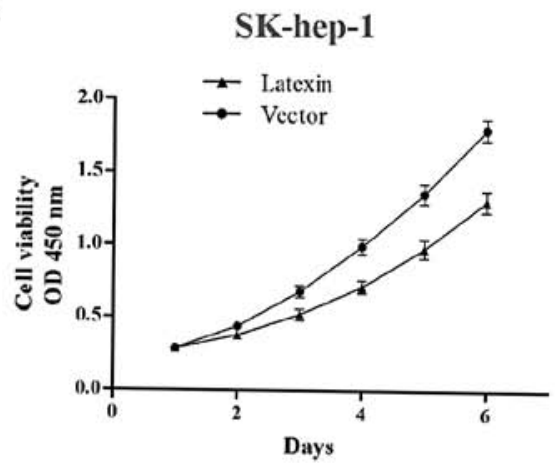

E

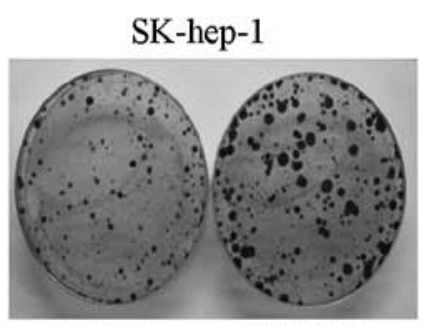

Latexin

Vector

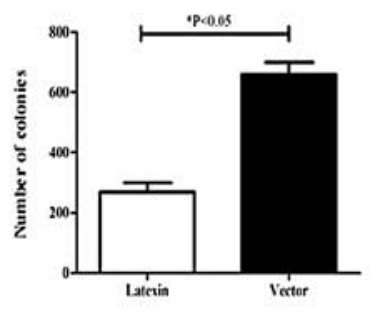

G

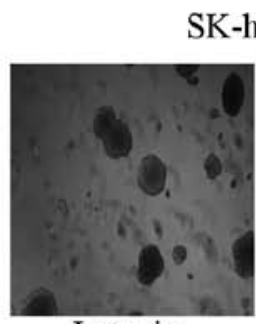

Latexin
SK-hep-1

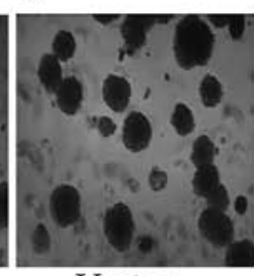

Vector

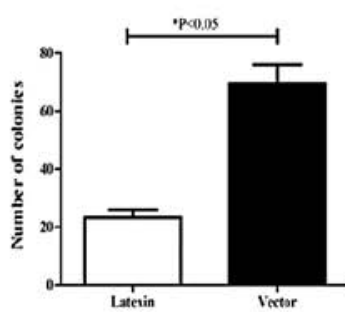

B

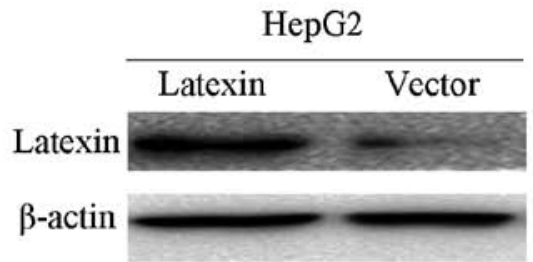

D

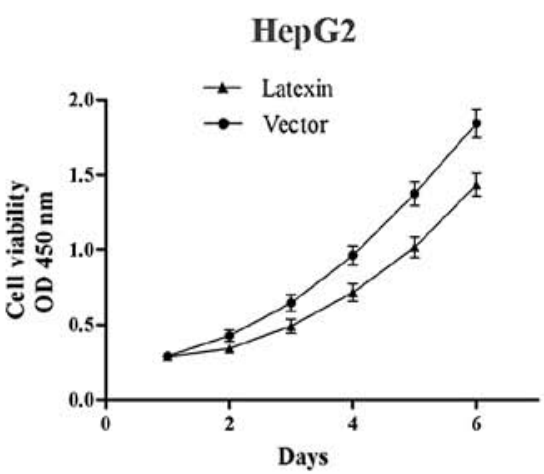

F
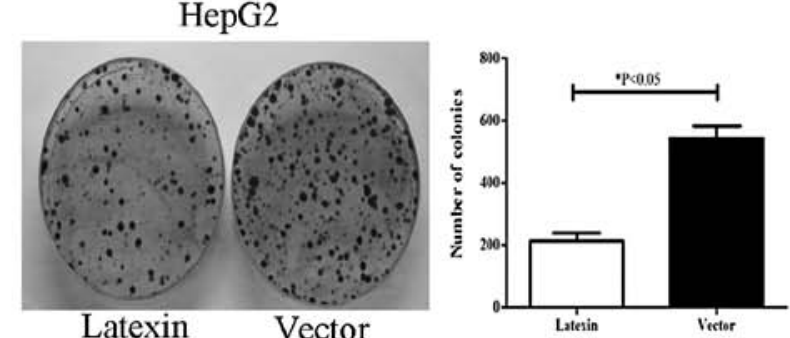

H
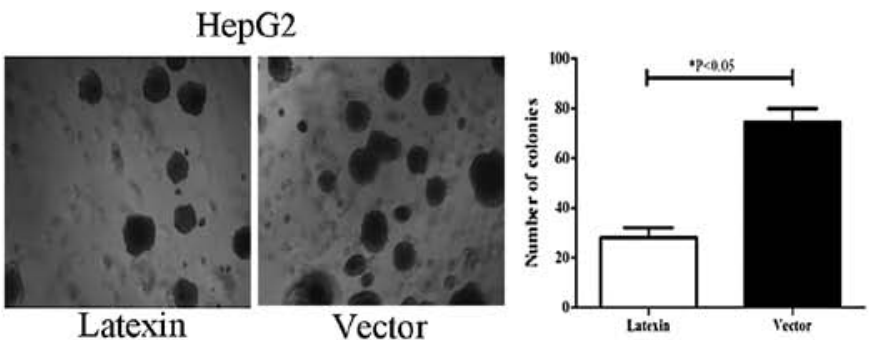

Figure 2. Overexpression of latexin inhibits proliferation and colony formation. (A and B) SK-hep-1 and HepG2 cells transfected with pcDNA3.1B-latexin stably increased latexin expression shown by western blotting. (C and D) Overexpression of latexin inhibits proliferation of SK-hep-1 and HepG2 cells $(\mathrm{P}<0.05)$. (E and F) Overexpression of latexin inhibits colony formation of SK-hep-1 and HepG2 cells $(\mathrm{P}<0.05)$. $(\mathrm{G}$ and $\mathrm{H}) \mathrm{Overexpression}$ of latexin inhibits soft agar colony formation of SK-hep-1 and HepG2 cells $(\mathrm{P}<0.05)$.

cells compared with the empty vector-transfected cells; the percentage of G0/G1 phase in the pcDNA3.1B-latexin group was increased by $13.57 \%\left({ }^{*} \mathrm{P}<0.05\right)$ at $24 \mathrm{~h}$. These results demonstrated that latexin overexpression may induce cell cycle arrest at G0/G1 phase. The opposite phenomenon was seen in YY-8103 cells when transfected with p-SUPERshRNA-latexin, as shown in Fig. 4C-E. Knockdown of latexin promoted the cell cycle transition from G0/G1 to $\mathrm{S}$ phase in YY-8103 cells compared with sh-NC-transfected cells; the percentage of $\mathrm{S}$ phase in the p-SUPER-shRNA-latexin group was increased by 14.01 and $12.11 \%\left({ }^{*} \mathrm{P}<0.05\right)$ at $24 \mathrm{~h}$. These results indicated that downregulation of latexin may facilitate cell entrance from the G0/G1 to the $\mathrm{S}$ phase, thus promoting cell proliferation.

Differential expression of latexin influences tumorigenesis in nude mice. The effects of differential latexin expression on the tumorigenic potential of liver cancer cells in vivo were also evaluated. SK-hep-1 cells overexpressing latexin and YY-8103 with downregulated latexin expression were injected subcutaneously into BALB/c nude mice $\left(500 \times 10^{6}\right.$ cells/mouse, 7 mice in each experimental group). Tumor size was measured every third day after injection. After 3 weeks, mice were sacrificed and photographed and the tumors were removed 
A

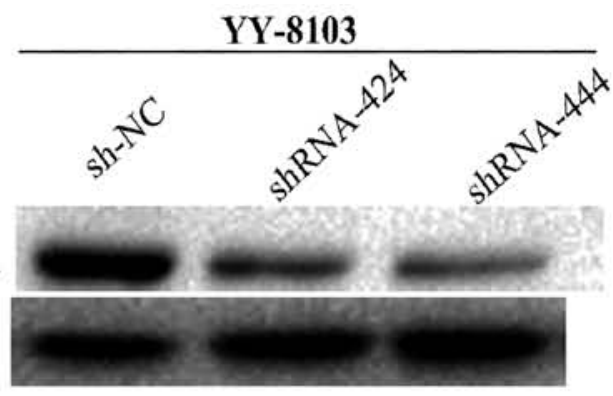

C

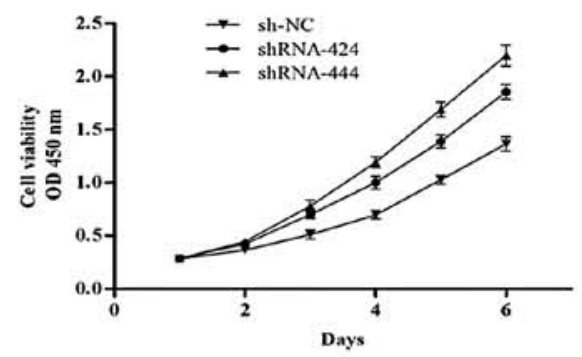

B

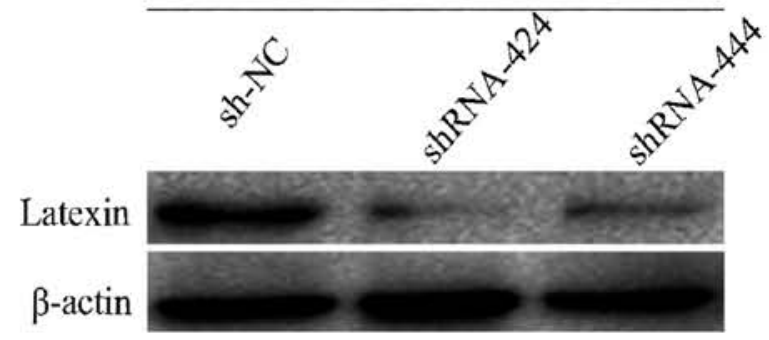

D

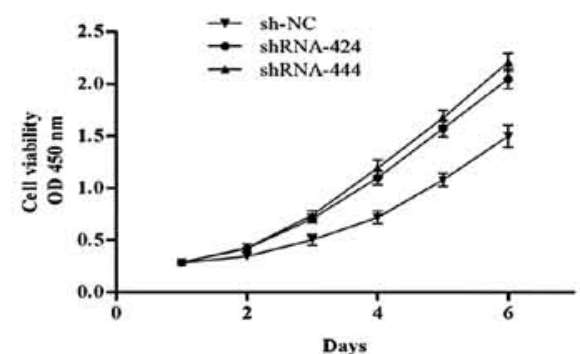

F

E
YY-8103

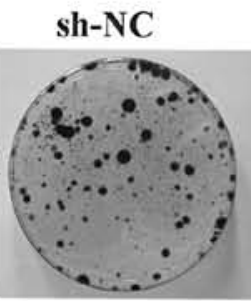

ShRNA-424
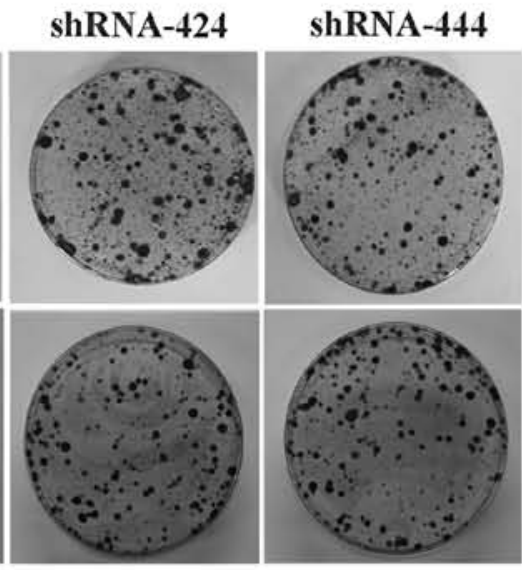

Focus
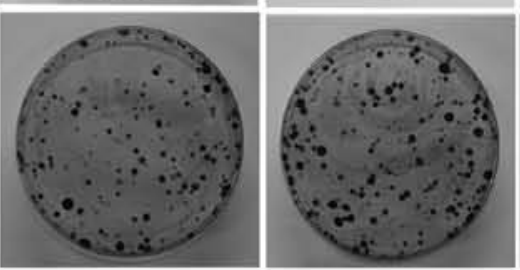

G
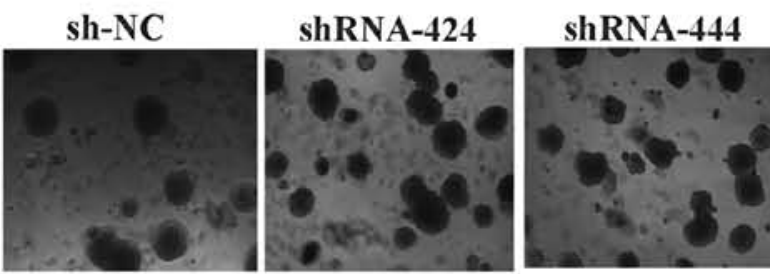

H
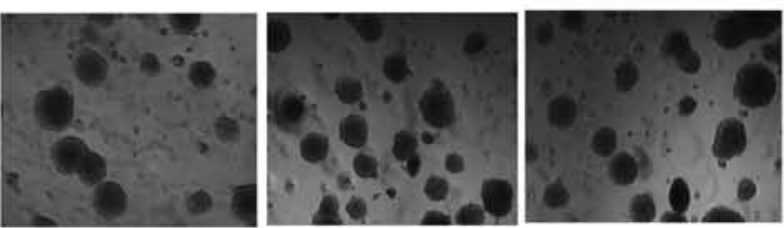
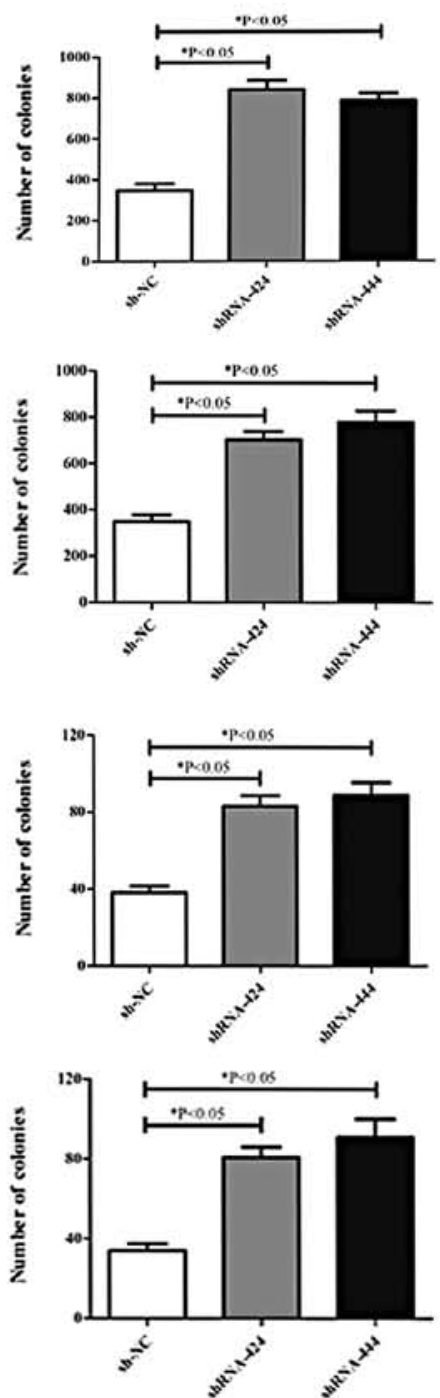

Figure 3. Knockdown of latexin promotes proliferation and colony formation. (A and B) YY-8103 and Focus cells transfected with p-SUPER-shRNA-latexin stably decreased latexin expression shown by western blotting. ( $\mathrm{C}$ and D) Knockdown of latexin promotes proliferation of $\mathrm{YY}-8103$ and Focus cells ( $\mathrm{P}<0.05)$. $\left(\mathrm{E}\right.$ and F) Knockdown of latexin promotes colony formation of YY-8103 and Focus cells $\left({ }^{*} \mathrm{P}<0.05\right)$. $(\mathrm{G}$ and $\mathrm{H}) \mathrm{Knockdown}$ of latexin promotes soft agar colony formation of YY-8103 and Focus cells $(\mathrm{P}<0.05)$. 
A

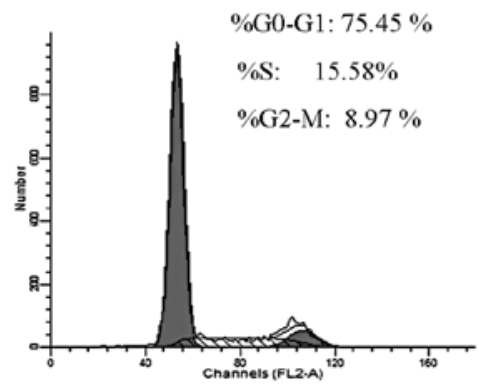

Latexin
B

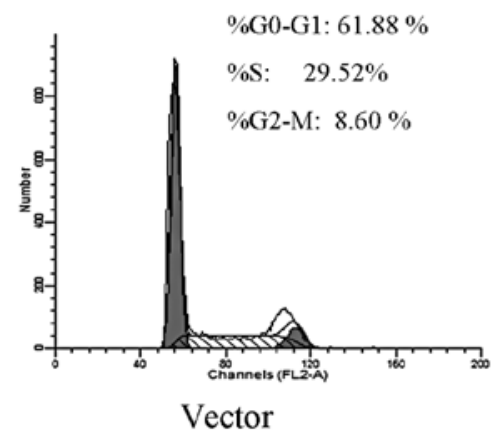

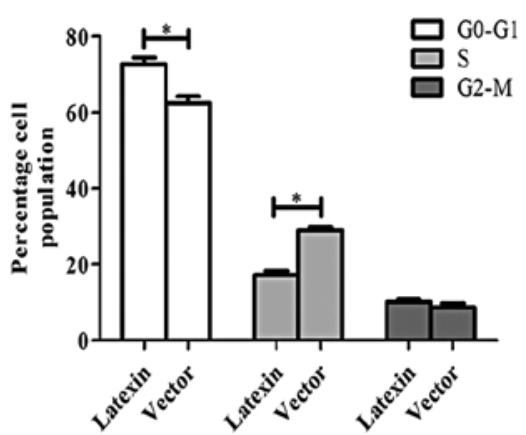

C

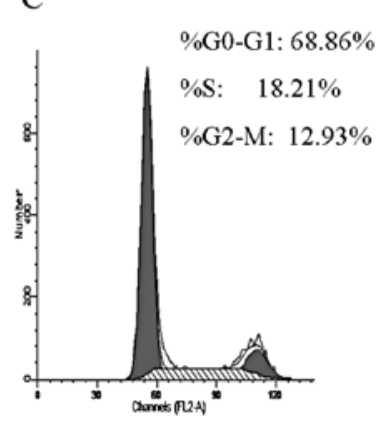

sh-NC
D

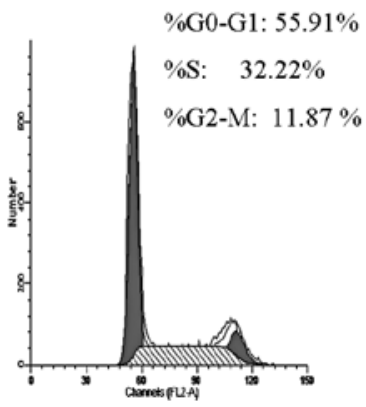

shRNA-424
E

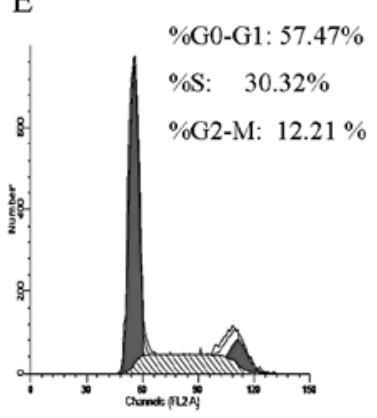

shRNA-444

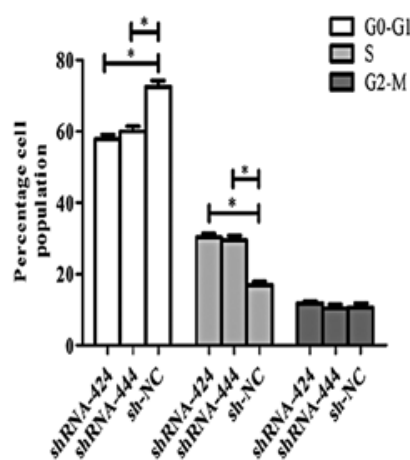

Figure 4. Representative flow cytometric analysis. (A and B) The number of cells in the G1 phase was significantly increased in pcDNA3.1B-latexin-transfected SK-hep-1 cells compared with empty vector-transfected cells ( $\left.{ }^{*} \mathrm{P}<0.05\right)$. (C-E) The number of cells in the $\mathrm{S}$ phase was significantly increased in p-SUPERshRNA-latexin-transfected YY-8103 cells compared with p-SUPER-sh-NC-transfected cells ( $\left.{ }^{*} \mathrm{P}<0.05\right)$.

and weighed. Compared to the mice injected with SK-hep-1 cells transfected with vector, the mice injected with SK-hep-1 cells overexpressing latexin displayed smaller tumors during the same time period, and the average tumor volumes and weights were significantly less than those in the control group $\left({ }^{* *} \mathrm{P}<0.01\right)$ (Fig. 5A, C and E). Compared to the mice injected with YY-8103 cells transfected with p-SUPER-sh-NC, the mice injected with YY-8103 cells and downregulated latexin showed a clear increased capacity for tumorigenesis $\left({ }^{* *} \mathrm{P}<0.01\right)$ (Fig. 5B, D and F). Taken together, these results strongly suggest that latexin acts as an inhibitor of tumor cell growth and tumorigenicity in vivo.

Overexpressed or silenced latexin influences the expression of cyclin pathway-related proteins. The protein expression data showed upregulation of p21Cip1, p27Kip1, p15INK4B and downregulation of cyclin D1 and cyclin E in the latexinoverexpressed cell line SK-hep-1 (Fig. 6A). Conversely, latexin-silencing via shRNA in the YY-8103 cell line showed downregulation of p21Cip1, p27Kip1, p15INK4B and upregulation of cyclin D1 and cyclin E (Fig. 6B).

\section{Discussion}

The silencing of latexin gene expression resulted in an enhanced capacity for colony formation and tumorigenicity in nude mice (22). A study by Greaves and Maley demonstrated that latexin may influence the crucial step in carcinogenesis (23). In addition, due to the close linkage both structurally and genetically with TIG1, we proposed that latexin may also act as a tumor suppressor. In the present study, we showed that latexin mRNA was downregulated in HCC samples and HCC-derived cell lines. Immunohistochemical analysis demonstrated that latexin was downregulated in the cytoplasm of HCC tissue. Of the HCC tissues tested, only $25.0 \%$ (15/60) were latexin-positive, while $60.0 \%$ of normal liver tissues (36/60) were latexin-positive. Colony formation assays and tumor xenografts in nude mice indicated a negative correlation between latexin expression and tumorigenesis of HCC.

To investigate the underlying molecular mechanism of latexin expression in negative control of tumor cell growth, we examined changes in the gene expression profile in response to differential latexin expression in SK-hep-1 and YY-8103 cells. We found that the variation in cellular proliferation was a result of cell cycle arrest at the G1 phase or cell cycle transition from the G0/G1 to the $S$ phase with differential expression of p21Cip1, p27Kip1, p15INK4B, cyclin D1 and cyclin E. These results were consistent with the observations that cell cycle progression is negatively controlled by CDKIs, such as p21Cip1, p27Kip1, p57Kip2 and the INK4 families (p15INK4B, p16INK4A, p18INK4C and p19INK4D), which are involved in cell cycle arrest at the G1 phase and have several functions as tumor suppressor genes (24), and that upregulation of p21Cip1 and/or p27Kip1 causes growth inhibition in various cancer 
A

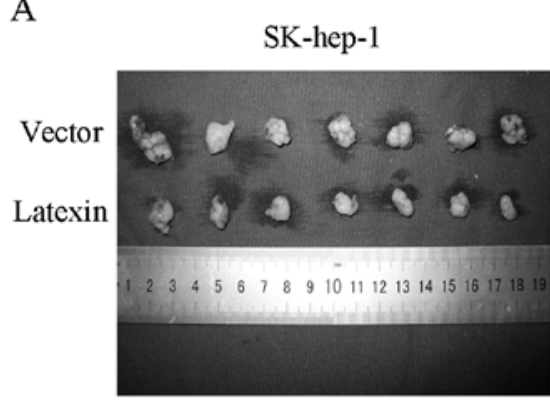

C

SK-hep-1

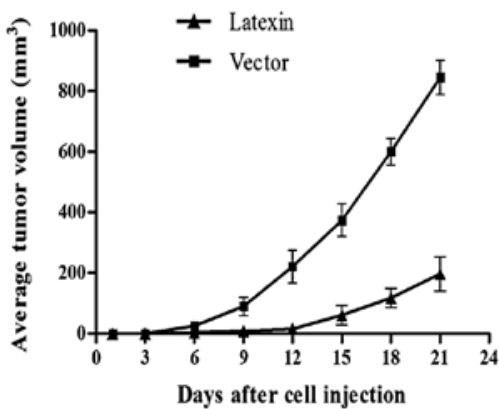

E

SK-hep-1

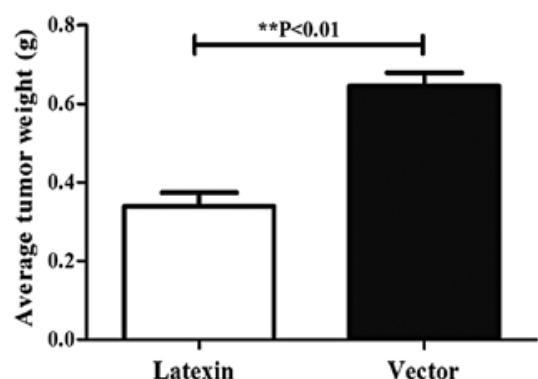

B

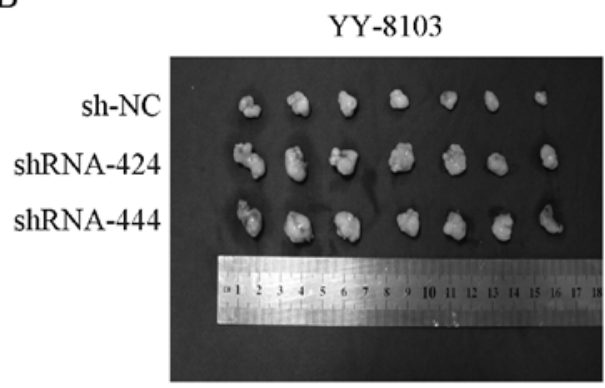

D

YY-8103

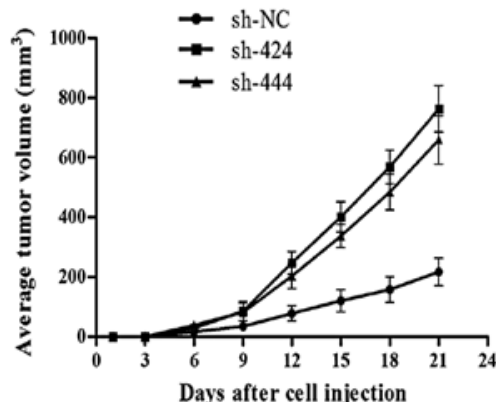

F

YY-8103

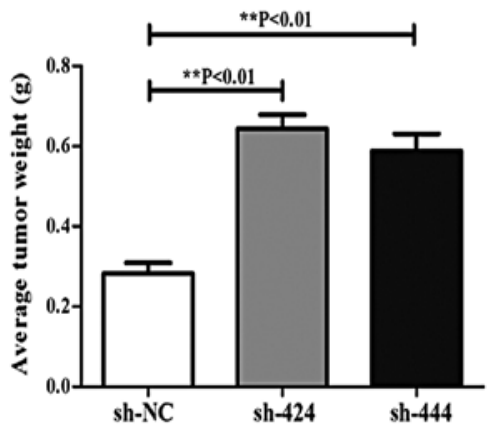

Figure 5. The effect of latexin expression on tumorigenesis in nude mice. (A and B) Tumors were excised 21 days after injection. (C and D) Tumor volume was measured every third day after injection $(\mathrm{P}<0.01)$. ( $\mathrm{E}$ and $\mathrm{F})$ The average weight of tumors in each group was assessed $\left.{ }^{* * *} \mathrm{P}<0.01\right)$.

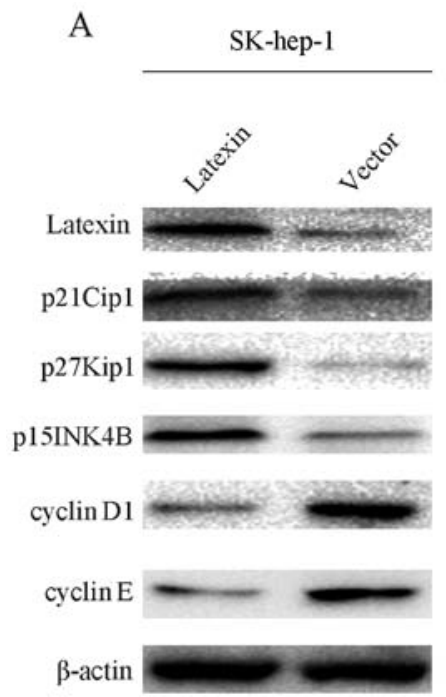

B

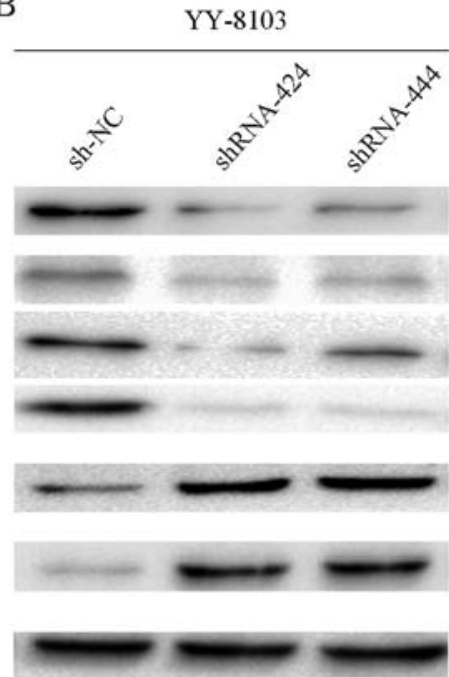

Figure 6. Western blot analysis of CDKIs (p21Cip1, p27Kip1 and p15INK4B), cyclin D1 and cyclin E. (A) Upregulation of p21Cip1, p27Kip1, p15INK4B and downregulation of cyclin D1 and cyclin E in latexin-overexpressed SK-hep-1 cells. (B) Downregulation of p21Cip1, p27Kip1, p15INK4B and upregulation of cyclin D1 and cyclin E in latexin-silenced YY-8103 cells. 
models (25-27). The INK4 families can bind to CDK4 and/or to CDK6 and inhibit the catalytic activity of the CDK/cyclin D complex (28-31). Cyclin D1, cyclin E, CDK4 and CDK6 are also critical regulators of G1 progression and G1-S transition (33). Inhibition of cyclin D1, cyclin E and CDK4 activation blocks G1-S transition in the cell cycle (32-35).

Our results showed that downregulation of latexin occurs frequently during liver carcinogenesis and that silencedlatexin may be associated with progression of HCC by preventing cessation of cell cycle progression at the G1 phase, through decreased expression of CDKIs and increased expression of cyclin D1 and cyclin E. Further studies are required to identify the detailed interaction between latexin and cell cycle regulators.

\section{Acknowledgements}

This study was supported by a grant from the Natural Science Foundation of China (81270483).

\section{References}

1. Llovet JM, Burroughs A and Bruix J: Hepatocellular carcinoma. Lancet 362: 1907-1917, 2003.

2. Farazi PA and DePinho RA: Hepatocellular carcinoma pathogenesis: from genes to environment. Nature Rev Cancer 6: 674-687, 2006.

3. El-Serag HB and Rudolph KL: Hepatocellular carcinoma: epidemiology and molecular carcinogenesis. Gastroenterology 132: 2557-2576, 2007.

4. Poon D, Anderson BO, Chen LT, Tanaka K, Lau WY, Van Cutsem E, et al: Management of hepatocellular carcinoma in Asia: consensus statement from the Asian Oncology Summit 2009. Lancet Oncol 10: 1111-1118, 2009.

5. Bruix J, Sherman M; Practice Guidelines Committee, American Association for the Study of Liver Diseases: Management of hepatocellular carcinoma. Hepatology 42: 1208-1236, 2005.

6. Coleman WB: Mechanism of human hepatocarcinogenesis. Curr Mol Med 3: 573-588, 2003

7. Caldwell S and Park SH: The epidemiology of hepatocellular cancer: from the perspectives of public health problem to tumor biology. J Gastroenterol 44 (Suppl 19): 96-101, 2009.

8. Bosch FX, Ribes J and Borràs J: Epidemiology of primary liver cancer. Semin Liver Dis 19: 271-285, 1999.

9. Tung-Ping Poon R, Fan ST and Wong J: Risk factors, prevention, and management of postoperative recurrence after resection of hepatocellular carcinoma. Ann Surg 232: 10-24, 2000.

10. Portolani N, Coniglio A, Ghidoni S, Giovanelli M, Benetti A, Tiberio GA and Giulini SM: Early and late recurrence after liver resection for hepatocellular carcinoma: prognostic and therapeutic implications. Ann Surg 243: 229-235, 2006.

11. Hatanaka Y, Uratani Y, Takiguchi-Hayashi K, Omori A, Sato K, Miyamoto $M$ and Arimatsu Y: Intracortical regionality represented by specific transcription for a novel protein, latexin. Eur J Neurosci 6: 973-982, 1994.

12. Liu Q, Yu L, Gao J, Fu Q, Zhang J, Zhang P, Chen J and Zhao S: Cloning, tissue expression pattern and genomic organization of latexin, a human homologue of rat carboxypeptidase A inhibitor. Mol Biol Rep 27: 241-246, 2000.

13. Aagaard A, Listwan P, Cowieson N, Huber T, Ravasi T, Wells CA, Flanagan JU, Kellie S, Hume DA, Kobe B and Martin JL: An inflammatory role for the mammalian carboxypeptidase inhibitor latexin: relationship to cystatins and the tumor suppressor TIG1. Structure 13: 309-317, 2005.
14. Pallarès I, Bonet R, García-Castellanos R, Ventura S, Avilés FX, Vendrell J and Gomis-Rüth FX: Structure of human carboxypeptidase A4 with its endogenous protein inhibitor, latexin. Proc Natl Acad Sci USA 102: 3978-3983, 2005.

15. Jing C, El-Ghany MA, Beesley C, Foster CS, Rudland PS, Smith P and Ke Y: Tazarotene-induced gene 1 (TIG1) expression in prostate carcinomas and its relationship to tumorigenicity. J Natl Cancer Inst 94: 482-490, 2002.

16. Liang Y, Jansen M, Aronow B, Geiger H and Van Zant G: The quantitative trait gene latexin influences the size of the hematopoietic stem cell population in mice. Nat Genet 39: 178-188, 2007.

17. Mitsunaga K, Kikuchi J, Wada T and Furukawa Y: Latexin regulates the abundance of multiple cellular proteins in hematopoietic stem cells. J Cell Physiol 227: 1138-1147, 2012.

18. Van Zant G and Liang Y: Natural genetic diversity as a means to uncover stem cell regulatory pathways. Ann NY Acad Sci 1176: 170-177, 2009.

19. Ke Y, Xu GW, Hagiwara K, Zhang JM, Ning T, Wang B, Su XL, Feng LY, Lu GR, Lu YY and Harris CC: Isolation and sequencing of the target genes induced by chemical carcinogen. Science in China (Series C) 26: 85-91, 1996.

20. Elledge SJ: Cell cycle checkpoints: preventing an identity crisis. Science 274: 1664-1672, 1996.

21. Sherr CJ: Cancer cell cycles. Science 274: 1672-1677, 1996.

22. Li Y, Basang Z, Ding H, et al: Latexin expression is downregulated in human gastric carcinomas and exhibits tumor suppressor potential. BMC Cancer 11: 111-121, 2011

23. Greaves $M$ and Maley CC: Clonal evolution in cancer. Nature 481: 306-313, 2012.

24. Porter PL, Malone KE, Heagerty PJ, et al: Expression of cell-cycle regulators $\mathrm{p} 27^{\mathrm{Kipl}}$ and cyclin $\mathrm{E}$, alone and in combination, correlate with survival in young breast cancer patients. Nat Med 3: 222-225, 1997

25. Eastham JA, Hall SJ, Sehgal I, et al: In vivo gene therapy with $p 53$ or $p 21$ adenovirus for prostate cancer. Cancer Res 55: 5151-5155, 1995.

26. Craig C, Wersto R, Kim M, et al: A recombinant adenovirus expressing p $27^{\mathrm{Kipl}}$ induces cell cycle arrest and loss of cyclin-Cdk activity in human breast cancer cells. Oncogene 14: 2283-2289, 1997.

27. Chen J, Willingham T, Shuford M, et al: Effects of ectopic overexpression of $\mathrm{p} 21^{\mathrm{WAF} / \mathrm{CIP1} 1}$ on aneuploidy and the malignant phenotype of human brain tumor cells. Oncogene 13: 1395-1403, 1996.

28. Hannon GJ and Beach D: p15 $5^{\mathrm{INK} 4 \mathrm{~B}}$ is a potential effector of TGF- $\beta$-induced cell cycle arrest. Nature 371: 257-261, 1994.

29. Guan KL, Jenkins CW, Li Y, et al: Growth suppression by p18, a p16INK4/MTS1- and p14INK4B/MTS2-related CDK6 inhibitor, correlates with wild-type pRb function. Genes Dev 8: 2939-2952, 1994.

30. Hirai H, Roussel MF, Kato JY, et al: Novel INK4 proteins, p19 and $\mathrm{p} 18$, are specific inhibitors of the cyclin D-dependent kinases CDK4 and CDK6. Mol Cell Biol 15: 2672-2681, 1995.

31. Chan FK, Zhang J, Cheng L, et al: Identification of human and mouse p19, a novel CDK4 and CDK6 inhibitor with homology to p16ink4. Mol Cell Biol 15: 2682-2688, 1995.

32. Sherr CJ and Roberts JM: CDK inhibitors: positive and negative regulators of G1-phase progression. Genes Dev 13: 1501-1512, 1999.

33. Kiviharju-af Hällström TM, Jäämaa S, Mönkkönen M, et al: Human prostate epithelium lacks Wee1A-mediated DNA damage-induced checkpoint enforcement. Proc Natl Acad Sci USA 104: 7211-7216, 2007.

34. Yoshida T, Tanaka S, Mogi A, et al: The clinical significance of Cyclin B1 and Weel expression in non-small-cell lung cancer. Ann Oncol 15: 252-256, 2004.

35. Quelle DE, Ashmun RA, Shurtleff SA, et al: Overexpression of mouse D-type cyclins accelerates G1 phase in rodent fibroblasts. Genes Dev 7: 1559-1571, 1993. 